\title{
Opening our worlds
}

\author{
KRISTINA SHEA \\ Virtual Product Development Group, Institute of Product Development, Technische Universität München, Garching, Germany
}

In his original article in 1950, Turing recognized the importance of defining the right world within which to explore the potential of artificial intelligence (AI) and thinking machines. "We hope that machines will eventually compete with men in all purely intellectual fields. But which are the best ones to start with? Even this is a difficult decision." Because AI EDAM focuses on AI for design, analysis, and manufacturing, this is a central question to the Journal. Where can AI have the greatest impact on design, analysis, and manufacture applications? Further, where can such applications provide interesting insights and issues that challenge the theories and methods applied?

In the early years of AI, and of AI EDAM, problems tended to be well defined, although still difficult, and the algorithms and systems to solve these problems were also typically closed. This was necessary to develop the first steps in this new research area, gain understanding, and show potential. However, now is the time to rethink the worlds we have defined in our research and open them up. I suggest some dimensions to think about.

The early writings in AI tended to take the human out of the loop, focusing on what machines could do autonomously and automatically. However, we have learned now that, most often, the human needs to be in the loop somewhere. Are we really seeking human-competitive results by a machine alone anyway or is the whole, that is, machine plus human, greater than the sum? Although initially perhaps thought to be "cheating," it has been shown that adding humans does not necessarily make problems easier, but it also allows us to open our worlds. In a similar direction, there is a trend from earlier closed systems (i.e., expert systems) to open support systems that are customizable by users so that they can incorporate evolving knowledge, because we know that knowledge always changes. Further, so that we can provide methods and tools that people want to use, we need to better understand humans and their cognitive processes during design, analysis, and manufacturing.

The next part of our world we need to think about is the scale of problems we define to tackle. Small-scale "toy" prob-

Reprint requests to: Kristina Shea, Virtual Product Development Group, Institute of Product Development, Technische Universität München, Boltzmannstrasse 15, Garching 85748, Germany. E-mail: kristina.shea@pe.mw. tum.de lems are no longer enough. Although they serve as good initial benchmarks, we need to move as a community to more complex, "industrial-strength" applications or as close as possible to validate our methods. Remember that this in turn can provide new insights about theories and methods! Even better are applications that are important economically and societally. Because of advances in computer science and available software, especially open source, we are also in the position to build better software prototypes and validate methods with users, such as students but better yet designers and engineers.

Another part of our world that we need to think about is the field or domain in which problems are defined. Most products today, whether consumer products, buildings, airplanes, cars, or machines, combine a complex mixture of disciplines that often include mechanical, structural, electrical, software, and, more recently, service aspects. Thus, the approaches and methods we are developing need to often move from single discipline approaches to tackle multiple disciplines. The complexity of their integration in design, analysis, and manufacture creates great challenges and thus great potential for new AI approaches.

Finally, when returning to reading some of the classic AI and AI EDAM references, I am always amazed at how clear the early ideas seemed and the impression that the fields of $\mathrm{AI}$, engineering, and cognitive sciences were more closely intertwined at the time. In the process of working out the details, the disciplines have diverged along the way. Now, there is a trend toward their reintegration and transfer of the latest theories, methods, software approaches, and understanding among disciplines, which is needed to tackle more complex application scenarios.

These are the challenges I suggest to think about to open your worlds. I look forward to the next 10 years of $A I$ $E D A M$ playing a key role in advancing this important, interdisciplinary field.

\section{REFERENCE}

Turing, A.M. (1950). Computing machinery and intelligence. Mind 49, 433 460. 